\title{
ON THE RICCI TENSOR OF SUBMANIFOLDS IN CONFORMAL KENMOTSU MANIFOLDS
}

\author{
Roghayeh ABDI and Esmaiel ABEDI \\ (Received 3 June 2016 and revised 16 October 2016)
}

\begin{abstract}
In this paper, we obtain several interesting results on submanifolds of conformal Kenmotsu manifolds. In addition to this we consider submanifolds of a conformal Kenmotsu manifold of which the Ricci tensor is parallel, Lie $\xi$-parallel or recurrent. We also present an illustration example of a three-dimensional conformal Kenmotsu manifold that is not Kenmotsu.
\end{abstract}

\section{Introduction}

Let $(M, J, g)$ be an almost Hermitian manifold of dimension $2 n$, where $J$ denotes the almost complex structure and $g$ the Hermitian metric. Then $(M, J, g)$ is called a locally conformal Kaehler manifold if for each point $p$ of $M$ there exists an open neighborhood $U$ of $p$ and a positive function $f_{U}$ on $U$ so that the local metric $g_{U}=\exp (-f) g_{\mid U}$ is Kaehlerian. If $U=M$, then the manifold $(M, J, g)$ is said to be a globally conformal Kaehler manifold. The 1 -form $\omega=d f$ is called the Lee form and its metrically equivalent vector field $\omega^{\sharp}=\operatorname{grad} f$, where $\sharp$ means the rising of the indices with respect to $g$, namely $g\left(X, \omega^{\sharp}\right)=\omega(X)$ for all $X$ tangent to $M$, is called the Lee vector field [9]. Submanifolds of locally conformal Kaehler manifolds with parallel Lee form have been studied by several authors (see, for instance, $[6,10,14])$.

In [11], Kenmotsu defined and studied a new class of almost contact manifolds called Kenmotsu manifolds.

We introduced conformal Kenmotsu manifolds by using an idea of globally conformal Kaehler manifolds (see [1] and [2]). Also we give an example of a conformal Kenmotsu manifold that is not Kenmotsu. Hence, the category of conformal Kenmotsu manifolds and Kenmotsu manifolds is completely different.

In [13], Kobayashi proved: let $M$ be a submanifold of a Kenmotsu manifold $\tilde{M}$ such that $\xi$ is tangent to $M$, then

$$
\nabla_{X} \xi=X-\eta(X) \xi, \quad h(X, \xi)=0, \quad R(X, Y) \xi=\eta(X) Y-\eta(Y) X
$$

for all $X$ and $Y$ tangent to $M$, where $\nabla, h$ and $R$ are the Riemannian connection, the second fundamental form and the curvature tensor of $M$, respectively.

In this paper, as a generalization of these results we state Lemmas 3.1, 3.2, 3.3, 3.4, 3.6 and 3.8 for a submanifold of a conformal Kenmotsu manifold.

2010 Mathematics Subject Classification: Primary 53C25, 53C40.

Keywords: Kenmotsu manifold; conformal Kenmotsu manifold; quasi-Einstein manifold.

(C) 2017 Faculty of Mathematics, Kyushu University 
A Riemannian manifold $\left(M^{n}, g\right)(n>2)$ is said to be an Einstein manifold if Ric $=$ $(r / n) g$ holds on $M$ where Ric and $r$ denote the Ricci tensor and the scalar curvature of $M$, respectively [3]. Einstein manifolds play an important role in Riemannian geometry as well as in physics. Every Einstein manifold belongs to the class of Riemannian manifolds $\left(M^{n}, g\right)$ realizing the following relation:

$$
\operatorname{Ric}(X, Y)=\operatorname{ag}(X, Y)+b A(X) A(Y),
$$

where $a$ and $b$ are smooth functions of which $b \neq 0$ and $A$ is a non-zero 1-form such that $g(X, U)=A(X)$ for each vector field $X$ and $U$ being a unit vector field which is called the generator of the manifold.

A non-flat Riemannian manifold $\left(M^{n}, g\right)(n>2)$ is said to be a quasi-Einstein manifold [7] if its Ricci tensor Ric is not identically zero and satisfies the condition (1.1). QuasiEinstein manifolds arose during the study of exact solutions of the Einstein field equations. For example, the Robertson-Walker space-time is a quasi-Einstein manifold [8]. So, quasiEinstein manifolds have some importance in general theory of relatively.

Motivated by the importance of Einstein and quasi-Einstein manifolds, in this paper we present the following problem:

Can we characterize submanifolds in a conformal Kenmotsu manifold with Lie $\xi$-parallel, recurrent or parallel Ricci tensor such that $\omega^{\sharp}$ is tangent (normal) to the submanifold?

Corresponding to this problem we give the following theorems.

THEOREM 1. Let $\bar{M}^{m}$ be a submanifold of a conformal Kenmotsu manifold $M$ with parallel or recurrent Ricci tensor such that $\omega^{\sharp}$ is normal to $\dot{M}$. Then $\dot{M}$ is an Einstein submanifold.

THEOREM 2. Let $\bar{M}^{m}$ be a submanifold of a conformal Kenmotsu manifold $M$ with parallel Ricci tensor such that $\omega^{\sharp}$ is tangent to $\dot{M}$. Then $\dot{M}$ is a quasi-Einstein submanifold.

THEOREM 3. There is not any submanifolds $\dot{M}^{m}$ of a conformal Kenmotsu manifold $M$ with Lie $\xi$-parallel Ricci tensor when $\omega^{\sharp}$ is tangent to $M$.

THEOREM 4. Let $\dot{M}^{m}$ be a submanifold of a conformal Kenmotsu manifold $M$ with recurrent Ricci tensor such that $\omega^{\sharp}$ is tangent to $M$. Then the Ricci tensor of Ḿ cannot be parallel.

The present paper is organized as follows. In Section 2, we recall some preliminary definitions on Kenmotsu manifolds, then we define conformal Kenmotsu manifolds. Furthermore, we give an example of a three-dimensional conformal Kenmotsu manifold that is not Kenmotsu. Section 3 gives some preliminary lemmas on submanifolds of a conformal Kenmotsu manifold tangent to $\xi$. In Section 4, we consider submanifolds of a conformal Kenmotsu manifold with parallel Ricci tensor such that $\omega^{\sharp}$ is normal (tangent) to the submanifold. We also give a non-existence theorem for submanifolds in conformal Kenmotsu manifolds with Lie $\xi$-parallel Ricci tensor when $\omega^{\sharp}$ is tangent to the submanifold. In Section 5, we consider submanifolds of a conformal Kenmotsu manifold with recurrent Ricci tensor, namely $\dot{\nabla} R i c=\alpha \otimes R i c$ where $\dot{\nabla}$ and Ric are the Riemannian connection and the Ricci tensor respectively and $\alpha$ is a 1 -form on the submanifold. We obtain values of $\alpha$ when $\omega^{\sharp}$ is normal (tangent) to submanifolds with recurrent Ricci tensor in conformal Kenmotsu manifolds. 


\section{Conformal Kenmotsu manifolds}

A $(2 n+1)$-dimensional differentiable manifold $M$ is an almost contact metric manifold, if it admits an almost contact metric structure $(\varphi, \xi, \eta, g)$ consisting of a tensor field $\varphi$ of type $(1,1)$, a vector field $\xi$, a 1-form $\eta$ and a Riemannian metric $g$ and satisfying following properties:

$$
\begin{aligned}
\varphi^{2}=-\mathrm{Id}+\eta \otimes \xi, \quad \eta(\xi)=1, \quad g(\varphi X, \varphi Y) & =g(X, Y)-\eta(X) \eta(Y), \\
\varphi \xi=0, \quad \eta \sigma \varphi=0, \quad \eta(X) & =g(X, \xi)
\end{aligned}
$$

for all vector fields $X, Y$ on $M,(2.1)$ is a consequence of the other conditions $[4, \mathbf{5}]$.

An almost contact metric manifold $\left(M^{2 n+1}, \varphi, \xi, \eta, g\right)$ is said to be a Kenmotsu manifold if the relation

$$
\left(\nabla_{X} \varphi\right) Y=-g(X, \varphi Y) \xi-\eta(Y) \varphi X
$$

holds on $M$, where $\nabla$ denotes the Riemannian connection of $g$. From (2.2) for a Kenmotsu manifold, we have

$$
\nabla_{X} \xi=X-\eta(X) \xi
$$

For a Kenmotsu manifold, we also have

$$
R(X, Y) \xi=\eta(X) Y-\eta(Y) X
$$

for all vector fields $X, Y$ tangent to $M$, where $R$ is the curvature tensor of $M$ (see [11]).

A $(2 n+1)$-dimensional smooth manifold $M$ with almost contact metric structure $(\varphi, \eta, \xi, g)$ is called a conformal Kenmotsu manifold if there exists a positive smooth function $f: M \rightarrow \mathbb{R}$ so that

$$
\tilde{g}=\exp (f) g, \quad \tilde{\xi}=(\exp (-f))^{1 / 2} \xi, \quad \tilde{\eta}=(\exp (f))^{1 / 2} \eta, \quad \tilde{\varphi}=\varphi
$$

is a Kenmotsu structure on $M$ (see [2]).

Let $M$ be a conformal Kenmotsu manifold, with $\tilde{\nabla}$ and $\nabla$ denoting the Riemannian connections of $M$ with respect to metrics $\tilde{g}$ and $g$, respectively. Using the Koszul formula, one simply can obtain the following relation between $\tilde{\nabla}$ and $\nabla$ :

$$
\tilde{\nabla}_{X} Y=\nabla_{X} Y+\frac{1}{2}\left\{\omega(X) Y+\omega(Y) X-g(X, Y) \omega^{\sharp}\right\}
$$

for all vector fields $X, Y$ on $M$, where $\omega(X)=g(\operatorname{grad} f, X)=X(f)$. Note that the vector field $\omega^{\sharp}=\operatorname{grad} f$ is called the Lee vector field of the conformal Kenmotsu manifold $M$. Then from $\eta(X)=g(X, \xi)$, we have the equality $\eta\left(\omega^{\sharp}\right)=\omega(\xi)$. Although $\omega\left(\omega^{\sharp}\right)=\left\|\omega^{\sharp}\right\|^{2}$, it is not necessarily $\left\|\omega^{\sharp}\right\|^{2}=1$ (unit vector field).

Assuming that $\tilde{R}$ and $R$ are, respectively, the curvature tensors of $(M, \varphi, \tilde{\eta}, \tilde{\xi}, \tilde{g})$ and $(M, \varphi, \eta, \xi, g)$. We have the following relation between $\tilde{R}$ and $R$ :

$$
\begin{aligned}
\exp (-f) \tilde{g}(\tilde{R}(X, Y) Z, W)= & g(R(X, Y) Z, W) \\
& +\frac{1}{2}\{B(X, Z) g(Y, W)-B(Y, Z) g(X, W) \\
& +B(Y, W) g(X, Z)-B(X, W) g(Y, Z)\} \\
& +\frac{1}{4}\left\|\omega^{\sharp}\right\|^{2}\{g(X, Z) g(Y, W)-g(Y, Z) g(X, W)\}
\end{aligned}
$$


for all vector fields $X, Y, Z, W$ on $M$ and $B$ satisfies

$$
B:=\nabla \omega-\frac{1}{2} \omega \otimes \omega \text {. }
$$

Obviously, $B$ is a symmetric tensor field of type $(0,2)$. On the other hand, from equations (2.2), (2.3) and (2.5), we get

$$
\begin{aligned}
\left(\nabla_{X} \varphi\right) Y= & (\exp (f))^{1 / 2}\{-g(X, \varphi Y) \xi-\eta(Y) \varphi X\} \\
& -\frac{1}{2}\left\{\omega(\varphi Y) X-\omega(Y) \varphi X+g(X, Y) \varphi \omega^{\sharp}-g(X, \varphi Y) \omega^{\sharp}\right\}, \\
\nabla_{X} \xi= & (\exp (f))^{1 / 2}\{X-\eta(X) \xi\}-\frac{1}{2}\left\{\omega(\xi) X-\eta(X) \omega^{\sharp}\right\}
\end{aligned}
$$

for all vector fields $X, Y$ on $M$.

\subsection{Example}

We give an example of a conformal Kenmotsu manifold but not necessarily a Kenmotsu manifold. We consider the three-dimensional manifold $M=\left\{(x, y, z) \in \mathbb{R}^{3}: x>0\right\}$ with the linearly independent vector fields

$$
e_{1}=x \frac{\partial}{\partial z}, \quad e_{2}=x \frac{\partial}{\partial y}, \quad e_{3}=-(\exp (x))^{1 / 2} x \frac{\partial}{\partial x} .
$$

Let $g$ be the Riemannian metric defined by

$$
\begin{aligned}
& g\left(e_{1}, e_{2}\right)=g\left(e_{1}, e_{3}\right)=g\left(e_{2}, e_{3}\right)=0, \\
& g\left(e_{1}, e_{1}\right)=g\left(e_{2}, e_{2}\right)=\exp (-x), \quad g\left(e_{3}, e_{3}\right)=1 .
\end{aligned}
$$

Let $\eta$ be the 1 -form defined by

$$
\eta\left(e_{3}\right)=1, \quad \eta\left(e_{2}\right)=0, \quad \eta\left(e_{1}\right)=0 .
$$

We define the $(1,1)$ tensor field $\varphi$ as $\varphi e_{1}=e_{2}, \varphi e_{2}=-e_{1}$ and $\varphi e_{3}=0$. Then using the linearity of $\varphi$ and $g$ we have

$$
\varphi^{2} X=-X+\eta(X) e_{3}, \quad g(\varphi X, \varphi Y)=g(X, Y)-\eta(X) \eta(Y)
$$

for all $X, Y$ on $M$. Thus, for $e_{3}=\xi,(\varphi, \xi, \eta, g)$ defines an almost contact metric structure on $M$.

On the other hand, we have

$$
\left[e_{1}, e_{2}\right]=0, \quad\left[e_{1}, e_{3}\right]=(\exp (x))^{1 / 2} e_{1}, \quad\left[e_{2}, e_{3}\right]=(\exp (x))^{1 / 2} e_{2} .
$$

The Riemannian connection $\nabla$ of the metric $g$ is given by

$$
\begin{aligned}
2 g\left(\nabla_{X} Y, Z\right)= & X g(Y, Z)+Y g(Z, X)-Z g(X, Y) \\
& -g(X,[Y, Z])+g(Y,[Z, X])+g(Z,[X, Y]),
\end{aligned}
$$

which is known as the Koszul formula. By using the Koszul formula, we obtain

$$
\begin{aligned}
& \nabla_{e_{1}} e_{1}=-\frac{1}{2}(\exp (-x))^{1 / 2}(x+2) e_{3}, \quad \nabla_{e_{1}} e_{2}=0, \\
& \nabla_{e_{1}} e_{3}=\frac{1}{2}(\exp (x))^{1 / 2}(x+2) e_{1}, \quad \nabla_{e_{2}} e_{1}=0, \\
& \nabla_{e_{2}} e_{2}=-\frac{1}{2}(\exp (-x))^{1 / 2}(x+2) e_{3}, \quad \nabla_{e_{2}} e_{3}=\frac{1}{2}(\exp (x))^{1 / 2}(x+2) e_{2}, \\
& \nabla_{e_{3}} e_{1}=\frac{1}{2}(\exp (x))^{1 / 2} x e_{1}, \quad \nabla_{e_{3}} e_{2}=\frac{1}{2}(\exp (x))^{1 / 2} x e_{2}, \quad \nabla_{e_{3}} e_{3}=0 .
\end{aligned}
$$


By the following contact transformation

$$
\tilde{g}=\exp (x) g, \quad \tilde{\xi}=(\exp (-x))^{1 / 2} \xi, \quad \tilde{\eta}=(\exp (x))^{1 / 2} \eta, \quad \tilde{\varphi}=\varphi,
$$

$(M, \tilde{\varphi}, \tilde{\xi}, \tilde{\eta}, \tilde{g})$ is a Kenmotsu manifold (see [15]). Hence, $(M, \varphi, \xi, \eta, g)$ is a conformal Kenmotsu manifold but is not Kenmotsu, since we have

$$
\left(\nabla_{X} \varphi\right) Y \neq-g(X, \varphi Y) \xi-\eta(Y) \varphi X
$$

for some vector fields $X, Y$ on $M$ (for instance, $\left.\left(\nabla_{e_{2}} \varphi\right) e_{1} \neq-g\left(e_{2}, \varphi e_{1}\right) \xi-\eta\left(e_{1}\right) \varphi e_{2}\right)$. By using above results, we can easily obtain the following:

$$
\begin{aligned}
& R\left(e_{1}, e_{2}\right) e_{2}=-\frac{1}{4}(x+2)^{2} e_{1}, \quad R\left(e_{2}, e_{3}\right) e_{3}=-\exp (x) e_{2}, \\
& R\left(e_{1}, e_{2}\right) e_{3}=0, \quad R\left(e_{1}, e_{3}\right) e_{3}=-\exp (x) e_{1}, \\
& R\left(e_{3}, e_{1}\right) e_{1}=-e_{3}, \quad R\left(e_{3}, e_{2}\right) e_{1}=0, \quad R\left(e_{3}, e_{1}\right) e_{2}=0, \\
& R\left(e_{2}, e_{1}\right) e_{1}=-\frac{1}{4}(x+2)^{2} e_{2}, \quad R\left(e_{3}, e_{2}\right) e_{2}=-e_{3} .
\end{aligned}
$$

In view of above relations, we get the following results:

$$
K\left(X, e_{3}\right)=-\exp (x), \quad K(X, Y)=-\frac{1}{4} \exp (x)(x+2)^{2}
$$

for all vector fields $X, Y$ orthogonal to $e_{3}$. Note that manifold $M$ with almost contact metric structure $(\tilde{\varphi}, \tilde{\xi}, \tilde{\eta}, \tilde{g})$ is a Kenmotsu manifold of constant curvature -1 (see [15]).

\section{Submanifolds of conformal Kenmotsu manifolds}

Let $(\dot{M}, g)$ be an $m$-dimensional submanifold into a $(2 n+1)$-dimensional conformal Kenmotsu manifold $(M, g)$. The Gauss and Weingarten formulas are given by

$$
\nabla_{X} Y=\dot{\nabla}_{X} Y+h(X, Y), \quad \nabla_{X} N=-A_{N} X+\nabla_{X}^{\perp} N
$$

for all vector fields $X, Y$ tangent to $\dot{M}$ and normal vector field $N$ on $\dot{M}$, where $\dot{\nabla}$ is the Riemannian connection of $\dot{M}$ determined by the induced metric $g$ and $\nabla^{\perp}$ is the normal connection on $T^{\perp} \dot{M}$ of $\dot{M}$. It is known that $g(h(X, Y), N)=g^{\prime}\left(A_{N} X, Y\right)$, where $A_{N}$ is the shape operator of $\dot{M}$ with respect to unit normal vector field $N$.

The Gauss equation is given by

$$
\begin{aligned}
\exp (-f) \tilde{g}(\tilde{R}(X, Y) Z, W)= & g(\dot{R}(X, Y) Z, W) \\
& -g(h(X, W), h(Y, Z))+g^{\prime}(h(Y, W), h(X, Z)) \\
& +\frac{1}{2}\{B(X, Z) \dot{g}(Y, W)-B(Y, Z) \dot{g}(X, W) \\
& +B(Y, W) g(X, Z)-B(X, W) g(Y, Z)\} \\
& +\frac{1}{4}\left\|\omega^{\sharp}\right\|^{2}\left\{\dot{g}(X, Z) \dot{g}(Y, W)-g^{\prime}(Y, Z) \dot{g}(X, W)\right\}
\end{aligned}
$$

for all $X, Y, Z, W$ tangent to $\dot{M}$ where $R$ is the curvature tensor of $\dot{M}$.

In this paper, we assume that $\xi$ is tangent to $\dot{M}$. 
LEMMA 3.1. Let $M$ be a submanifold of a conformal Kenmotsu manifold $M$ such that $\omega^{\sharp}$ is normal to $M$. Then

$$
\begin{aligned}
B(X, Y) & =-\omega(h(X, Y)), \\
h(X, \xi) & =\frac{1}{2} \eta(X) \omega^{\sharp}, \\
\dot{\nabla}_{X} \xi & =(\exp (f))^{1 / 2}\{X-\eta(X) \xi\}
\end{aligned}
$$

for all vector fields $X, Y$ tangent to $M$.

Proof. From (2.7) we have

$$
B(X, Y)=\left(\nabla_{X} \omega\right) Y-\frac{1}{2} \omega(X) \omega(Y)=\nabla_{X}(\omega(Y))-\omega\left(\nabla_{X} Y\right)-\frac{1}{2} \omega(X) \omega(Y)
$$

for all $X, Y$ tangent to $\dot{M}$. Since $\omega^{\sharp}$ is normal to $\dot{M}$, the above equation can be written as

$$
B(X, Y)=-\omega\left(\nabla_{X} Y\right)
$$

for all $X, Y$ on $\dot{M}$. Then by the use of the Gauss formula we obtain (3.2).

Taking $Y=\xi$ in the Gauss formula and using (2.9) we have

$$
\dot{\nabla}_{X} \xi+h(X, \xi)=\nabla_{X} \xi=(\exp (f))^{1 / 2}\{X-\eta(X) \xi\}-\frac{1}{2}\left\{\omega(\xi) X-\eta(X) \omega^{\sharp}\right\}
$$

for each $X$ tangent to $\dot{M}$. Since $\omega^{\sharp}$ is normal to $\dot{M}$, we compare the tangential part and the normal part in the above equation. Then we obtain (3.3) and (3.4).

LEMMA 3.2. Let $M$ be a submanifold of a conformal Kenmotsu manifold $M$ such that $\omega^{\sharp}$ is tangent to $M$. Then

$$
\begin{aligned}
B(X, Y) & =\dot{g}\left(\dot{\nabla}_{X} \omega^{\sharp}, Y\right)-\frac{1}{2} \omega(X) \omega(Y), \\
h(X, \xi) & =0, \\
\dot{\nabla}_{X} \xi & =(\exp (f))^{1 / 2}\{X-\eta(X) \xi\}-\frac{1}{2}\left\{\omega(\xi) X-\eta(X) \omega^{\sharp}\right\}
\end{aligned}
$$

for all vector fields $X, Y$ tangent to $M$.

Proof. Similarly to Lemma 3.1, equations (3.5), (3.6) and (3.7) are immediate results from (2.7), (2.9) and the Gauss formula.

LEMMA 3.3. Let $M$ be a submanifold of a conformal Kenmotsu manifold $M$ such that $\omega^{\sharp}$ is normal to $M$. Then

$$
\begin{aligned}
& \dot{R}(X, Y) \xi=\exp (f)\{\eta(X) Y-\eta(Y) X\}, \\
& \dot{R}(X, \xi) Y=\exp (f)\{g(X, Y) \xi-\eta(Y) X\}
\end{aligned}
$$

for all vector fields $X, Y$ tangent to $M$.

Proof. Equation (3.8) follows from (2.4), (3.1), (3.2) and (3.3). From (3.8) and the symmetric property of $R$ we get (3.9). 
LEMMA 3.4. Let $M$ be a submanifold of a conformal Kenmotsu manifold $M$ such that $\omega^{\sharp}$ is tangent to $M$. Then

$$
\begin{aligned}
\dot{R}(X, Y) \xi= & \left(\exp (f)-\frac{1}{4}\left\|\omega^{\sharp}\right\|^{2}\right)\{\eta(X) Y-\eta(Y) X\} \\
& -\frac{1}{2}\left\{\eta\left(\dot{\nabla}_{X} \omega^{\sharp}\right) Y-\frac{1}{2} \omega(X) \omega(\xi) Y-\eta\left(\dot{\nabla}_{Y} \omega^{\sharp}\right) X+\frac{1}{2} \omega(Y) \omega(\xi) X\right. \\
& \left.+\eta(X) \dot{\nabla}_{Y} \omega^{\sharp}-\frac{1}{2} \eta(X) \omega(Y) \omega^{\sharp}-\eta(Y) \dot{\nabla}_{X} \omega^{\sharp}+\frac{1}{2} \eta(Y) \omega(X) \omega^{\sharp}\right\}
\end{aligned}
$$

for all vector fields $X, Y$ tangent to $\dot{M}$.

Proof. Equations (2.4), (3.1), (3.5) and (3.6) yield (3.10).

COROLlARY 3.5. Let $M$ be a submanifold of a conformal Kenmotsu manifold $M$ such that $\omega^{\sharp}$ is tangent to $\dot{M}$. If $\omega^{\sharp}$ is parallel on $\dot{M}$, then

$$
\begin{aligned}
\dot{R}(X, Y) \xi= & \left(\exp (f)-\frac{1}{4}\left\|\omega^{\sharp}\right\|^{2}\right)\{\eta(X) Y-\eta(Y) X\} \\
& +\frac{1}{4}\left\{\omega(X) \omega(\xi) Y-\omega(Y) \omega(\xi) X+\eta(X) \omega(Y) \omega^{\sharp}-\eta(Y) \omega(X) \omega^{\sharp}\right\}, \\
\dot{R}(X, \xi) Y= & \left(\exp (f)-\frac{1}{4}\left\|\omega^{\sharp}\right\|^{2}\right)\{\dot{g}(X, Y) \xi-\eta(Y) X\} \\
& +\frac{1}{4}\left\{\omega(\xi) g(X, Y) \omega^{\sharp}-\omega(\xi) \omega(Y) X+\omega(X) \omega(Y) \xi-\omega(X) \eta(Y) \omega^{\sharp}\right\}
\end{aligned}
$$

for all vector fields $X, Y$ tangent to $\dot{M}$.

Proof. Equation (3.11) is an immediate result of (3.10). We obtain (3.12) by (3.11) and the symmetric property of $R$.

LEMMA 3.6. Let $\dot{M}^{m}$ be a submanifold of a conformal Kenmotsu manifold M such that $\omega^{\sharp}$ is normal to $M$. Then

$$
\operatorname{Ric}(X, \xi)=-(m-1) \exp (f) \eta(X)
$$

for each vector field $X$ tangent to $\dot{M}$ where Ric denotes the Ricci tensor of $\dot{M}$.

Proof. Suppose that $\left\{e_{i}, \xi \mid i=1, \ldots, m-1\right\}$ is an orthonormal basis on $\dot{M}$. The definition of the Ricci tensor in $\dot{M}^{m}$ yields

$$
\operatorname{Ric}(X, \xi)=\sum_{i=1}^{m-1} g\left(\hat{R}\left(e_{i}, X\right) \xi, e_{i}\right) .
$$

By using (3.8), we get (3.13).

COROLlARY 3.7. Let $\bar{M}^{m}(m>1)$ be a submanifold of a conformal Kenmotsu manifold $M$ such that $\omega^{\sharp}$ is normal to $\dot{M}$. Then $M$ cannot be Ricciflat.

Proof. Let $M$ be Ricci flat. Putting $X=\xi$ in (3.13), one simply get $\exp (f)=0$ that is a contradiction.

LEMMA 3.8. Let $\dot{M}^{m}$ be a submanifold of a conformal Kenmotsu manifold $M$ such that $\omega^{\sharp}$ is tangent to $\dot{M}$. If $\omega^{\sharp}$ is parallel on $\dot{M}$, then

$$
\begin{aligned}
\operatorname{Ric}(X, \xi)= & \left(-(m-1) \exp (f)+\frac{1}{4}(m-2)\left\|\omega^{\sharp}\right\|^{2}\right) \eta(X) \\
& -\frac{1}{4}(m-2) \omega(\xi) \omega(X)
\end{aligned}
$$

for each vector field $X$ tangent to $M$. 
Proof. Suppose that $\left\{e_{i}, \xi \mid i=1, \ldots, m-1\right\}$ is an orthonormal basis on $\dot{M}$. The definition of the Ricci tensor in $\dot{M}^{m}$ implies that

$$
\operatorname{Ric}(X, \xi)=\sum_{i=1}^{m-1} g\left(\hat{R}\left(e_{i}, X\right) \xi, e_{i}\right) .
$$

By using (3.11), we get

$$
\begin{aligned}
\operatorname{Ric}(X, \xi)= & \left(-(m-1) \exp (f)+\frac{1}{4}(m-1)\left\|\omega^{\sharp}\right\|^{2}\right) \eta(X)-\frac{1}{4}(m-1) \omega(\xi) \omega(X) \\
& +\frac{1}{4} \sum_{i=1}^{m-1}\left\{\omega\left(e_{i}\right) \omega(\xi) g\left(X, e_{i}\right)-\eta(X) \omega\left(e_{i}\right)^{2}\right\} .
\end{aligned}
$$

On the other hand, we have

$$
\dot{g}(X, Y)=\sum_{i=1}^{m-1} g\left(X, e_{i}\right) \dot{g}\left(Y, e_{i}\right)+g^{\prime}(X, \xi) g(Y, \xi)
$$

for all vector fields $X, Y$ on $\dot{M}$. Then equations (3.15) and (3.16) yield (3.14).

From now on, we suppose that the Lee vector field $\omega^{\sharp}$ is non-zero.

LEMMA 3.9. Let $M$ be a submanifold of a conformal Kenmotsu manifold $M$ such that $\omega^{\sharp}$ is tangent to $\dot{M}$. If $\omega^{\sharp}$ is parallel on $\dot{M}$, then

$$
\begin{gathered}
\omega(\xi) \neq 0, \\
(\exp (f))^{1 / 2}-\frac{1}{2} \omega(\xi) \neq 0, \\
\alpha \exp (f)+\beta\left(\left\|\omega^{\sharp}\right\|^{2}-\omega(\xi)^{2}\right) \neq 0 .
\end{gathered}
$$

In (3.19), $\alpha$ and $\beta$ are some non-zero constants.

Proof. The proof of the relations (3.17), (3.18) and (3.19) are given by contradiction.

Suppose $\omega(\xi)=0$. Taking the covariant differentiation of $\omega(\xi)=0$ with respect to $\xi$ and using $\nabla \omega^{\sharp}=0$, we obtain

$$
\stackrel{g}{g}\left(\dot{\nabla}_{\xi} \xi, \omega^{\sharp}\right)=0 .
$$

Using (3.7) in the above equation, we get

$$
\left\|\omega^{\sharp}\right\|^{2}=\omega(\xi)^{2} .
$$

Since we suppose that $\omega(\xi)=0$, from the above equation it follows that $\left\|\omega^{\sharp}\right\|^{2}=0$ which contradicts the hypothesis $\omega^{\sharp} \neq 0$. Hence (3.17) holds on $\dot{M}$.

Now, we assume

$$
(\exp (f))^{1 / 2}-\frac{1}{2} \omega(\xi)=0 .
$$

Taking the covariant differentiation of (3.20) along vector field $\xi$, we have

$$
\frac{1}{2} \omega(\xi)(\exp (f))^{1 / 2}-\frac{1}{2} \xi(\omega(\xi))=0 .
$$

Using (3.20) and $\dot{\nabla} \omega^{\sharp}=0$ in (3.21), we get $2 \dot{g}\left(\dot{\nabla}_{\xi} \xi, \omega^{\sharp}\right)=\omega(\xi)^{2}$. Then by the use of (3.7) in $2 g\left(\dot{\nabla}_{\xi} \xi, \omega^{\sharp}\right)=\omega(\xi)^{2}$, we find $2 \omega(\xi)^{2}=\left\|\omega^{\sharp}\right\|^{2}$. As $\omega^{\sharp}$ is parallel on $\dot{M}$, it follows that 
$\left\|\omega^{\sharp}\right\|^{2}$ is constant on $\dot{M}$. Thus, $\omega(\xi)^{2}$ is constant on $\dot{M}$. Then from $(3.21)$ we have $\omega(\xi)=0$ which is a contradiction in view of (3.17).

Finally, we suppose

$$
\alpha \exp (f)+\beta\left(\left\|\omega^{\sharp}\right\|^{2}-\omega(\xi)^{2}\right)=0 .
$$

Taking the covariant differentiation of (3.22) along vector field $\xi$ and using $\dot{\nabla} \omega^{\sharp}=0$, we find

$$
\alpha \omega(\xi) \exp (f)-2 \beta \omega(\xi) \dot{g}\left(\dot{\nabla}_{\xi} \xi, \omega^{\sharp}\right)=0 .
$$

Making use of (3.7) and (3.17) in the above equation, we get

$$
\alpha \exp (f)+\beta\left(\omega(\xi)^{2}-\left\|\omega^{\sharp}\right\|^{2}\right)=0 .
$$

Summing (3.22) to (3.23), we have $\alpha \exp (f)=0$ which is a contradiction.

Hence (3.17), (3.18) and (3.19) hold on $\dot{M}$.

COROLlary 3.10. Let $\bar{M}^{m}(m>1)$ be a submanifold of a conformal Kenmotsu manifold $M$ such that $\omega^{\sharp}$ is tangent to $M$. Suppose that $\omega^{\sharp}$ is parallel on $\dot{M}$. Then $\dot{M}$ cannot be Ricci flat.

Proof. Suppose $M$ is Ricci flat. Putting $X=\omega^{\sharp}$ in (3.14), we obtain

$$
(m-1) \exp (f) \omega(\xi)=0 .
$$

Using (3.17) in the above equation, we get $\exp (f)=0$ that is a contradiction.

\section{Submanifolds with parallel Ricci tensor}

The notion of Einstein manifolds and quasi-Einstein manifolds was introduced in [3] and [7], respectively.

Therefore, a submanifold $\left(\dot{M}^{m}, g\right)(m>2)$ of a conformal Kenmotsu manifold $(M, g)$ is an Einstein manifold if its Ricci tensor Ric satisfies the condition Ric $=\lambda \dot{g}$, where $\lambda$ is a non-zero constant.

Moreover, a submanifold $\left(\bar{M}^{m}, g\right)(m>2)$ of a conformal Kenmotsu manifold $(M, g)$ is a quasi-Einstein manifold if the condition

$$
\operatorname{Ric}(X, Y)=a^{g}(X, Y)+b A(X) A(Y)
$$

is fulfilled on $\bar{M}$, where $a, b$ are smooth functions of which $b \neq 0$ and $A$ is non-zero 1 -form such that

$$
g(X, U)=A(X)
$$

for all vector fields $X$ and $U$ being a unit vector field which is called the generator of $M$.

In [13], Kobayashi showed that a submanifold of a Kenmotsu manifold has parallel second fundamental form if and only if the submanifold is totally geodesic. As a generalization of this result, we consider submanifolds of a conformal Kenmotsu manifold with parallel Ricci tensor.

Now we state the following theorems.

THEOREM 4.1. Let $\bar{M}^{m}(m>2)$ be a submanifold of a conformal Kenmotsu manifold $M$ with parallel Ricci tensor such that $\omega^{\sharp}$ is normal to $\dot{M}$. Then $\dot{M}$ is an Einstein submanifold. 
Proof. Taking the covariant differentiation of (3.13) with respect to vector field $Y$, we obtain

$$
\operatorname{Ric}\left(X, \dot{\nabla}_{Y} \xi\right)=-(m-1) \exp (f) \dot{g}\left(X, \dot{\nabla}_{Y} \xi\right) .
$$

Making use of (3.4) and (3.13) in the above equation, it follows that

$$
\operatorname{Ric}(X, Y)=-(m-1) \exp (f) g(X, Y)
$$

for all $X, Y$ on $\dot{M}$. Hence, $\dot{M}$ is an Einstein submanifold.

THEOREM 4.2. Let $\bar{M}^{m}(m>2)$ be a submanifold of a conformal Kenmotsu manifold $M$ with parallel Ricci tensor such that $\omega^{\sharp}$ is tangent to $\dot{M}$. Then $\dot{M}$ is a quasi-Einstein submanifold.

Proof. Suppose that $\omega^{\sharp}$ is parallel on $\dot{M}$. Taking the covariant differentiation of (3.14) and using (3.7), we have

$$
\begin{aligned}
& \left((\exp (f))^{1 / 2}-\frac{1}{2} \omega(\xi)\right)\{\operatorname{Ric}(X, Y)-(-(m-1) \exp (f) \\
& \left.\left.\quad+\frac{1}{4}(m-2)\left\|\omega^{\sharp}\right\|^{2}\right) g(X, Y)+\frac{1}{4}(m-2) \omega(X) \omega(Y)\right\}=0
\end{aligned}
$$

for all vector fields $X, Y$ orthogonal to $\xi$. By the use of (3.18) in (4.3) we get

$$
\begin{aligned}
\operatorname{Ric}(X, Y)= & \left\{-(m-1) \exp (f)+\frac{1}{4}(m-2)\left\|\omega^{\sharp}\right\|^{2}\right\} g(X, Y) \\
& -\frac{1}{4}(m-2) \omega(X) \omega(Y),
\end{aligned}
$$

where $X, Y$ are orthogonal to $\xi$. We get (4.4) for all vector fields $X, Y$ in view of (3.14). Since $\omega^{\sharp} \neq 0$, (4.4) can be written as

$$
\begin{aligned}
\operatorname{Ric}(X, Y)= & \left\{-(m-1) \exp (f)+\frac{1}{4}(m-2)\left\|\omega^{\sharp}\right\|^{2}\right\} g(X, Y) \\
& -\frac{1}{4}(m-2)\left\|\omega^{\sharp}\right\|^{2} g\left(X, \frac{\omega^{\sharp}}{\left\|\omega^{\sharp}\right\|}\right) g\left(Y, \frac{\omega^{\sharp}}{\left\|\omega^{\sharp}\right\|}\right)
\end{aligned}
$$

for all $X, Y$ tangent to $\dot{M}$. Hence, by (4.1) $\dot{M}$ is a quasi-Einstein submanifold.

THEOREM 4.3. There is not any submanifolds $\bar{M}^{m}(m>1)$ of a conformal Kenmotsu manifold $M$ with Lie $\xi$-parallel Ricci tensor when $\omega^{\sharp}$ is tangent to $M$.

Proof. Since the Ricci tensor of $\dot{M}$ is Lie $\xi$-parallel, we can write

$$
0=L_{\xi} \operatorname{Ric}(X, \xi)-\operatorname{Ric}\left(L_{\xi} X, \xi\right)
$$

for each $X$ on $\dot{M}$ where $L_{\xi}$ is the Lie derivative. Let $\omega^{\sharp}$ be parallel on $\dot{M}$. Putting (3.14) into the above equation, we have

$$
\begin{aligned}
0= & \left\{-(m-1) \exp (f)+\frac{1}{4}\left\|\omega^{\sharp}\right\|^{2}(m-2)\right\}\left(L \xi g^{\prime}\right)(X, \xi) \\
& -(m-1) \exp (f) \omega(\xi) \eta(X)+\frac{1}{4}(m-2) \omega(\xi) g\left(X, \dot{\nabla}_{\omega^{\sharp}} \xi\right) .
\end{aligned}
$$

Then by the use of (3.7), we get

$$
\begin{aligned}
0= & \left\{-(m-1) \exp (f)+\frac{1}{4}\left\|\omega^{\sharp}\right\|^{2}(m-2)\right\}\left(L_{\xi} g\right)(X, \xi) \\
& -(m-1) \exp (f) \omega(\xi) \eta(X)+\frac{1}{4}(m-2)(\exp (f))^{1 / 2} \omega(\xi)(\omega(X)-\omega(\xi) \eta(X))
\end{aligned}
$$


for each $X$ on $\dot{M}$. Taking $X=\xi$ in the above equation, we obtain

$$
\exp (f) \omega(\xi)=0 .
$$

Making use of relation (3.17) in (4.5), we have $\exp (f)=0$ that is a contradiction. Hence, the proof of the theorem is completed.

\section{Submanifolds with recurrent Ricci tensor}

The notion of a recurrent tensor field of type $(r, s)$ on a differentiable manifold $M$ with a linear connection $\nabla$ was introduced in [12] and [16]. A non-zero tensor field $K$ of type $(r, s)$ on $M$ is recurrent if there is a 1 -form $\alpha$ such that

$$
\nabla K=\alpha \otimes K .
$$

Therefore, a submanifold $\bar{M}$ of a conformal Kenmotsu manifold is said to be with recurrent Ricci tensor if there exists a 1 -form $\alpha$ on $\dot{M}$ such that

$$
\left(\dot{\nabla}_{X} \operatorname{Ric}\right)(Y, Z)=\alpha(X) \operatorname{Ric}(Y, Z)
$$

for all vector fields $X, Y, Z$ on $\dot{M}$ where Ric denotes the Ricci tensor of $\dot{M}$.

Now, we state the following results.

LEMMA 5.1. Let $\dot{M}^{m}(m>1)$ be a submanifold of a conformal Kenmotsu manifold $M$ with recurrent Ricci tensor such that $\omega^{\sharp}$ is normal to $\dot{M}$. Then the Ricci tensor of $\dot{M}$ is parallel.

Proof. Taking $X=\xi$ in (3.13), we have

$$
\operatorname{Ric}(\xi, \xi)=-(m-1) \exp (f) .
$$

Taking covariant differentiation of (5.2) along vector field $X$, we obtain

$$
\left(\dot{\nabla}_{X} \operatorname{Ric}\right)(\xi, \xi)+2 \operatorname{Ric}\left(\dot{\nabla}_{X} \xi, \xi\right)=-(m-1) X(f) \exp (f) .
$$

Since Ric is recurrent and $X(f)=\omega(X)$, from (5.1) the above equation can be written as

$$
\alpha(X) \operatorname{Ric}(\xi, \xi)+2 \operatorname{Ric}\left(\dot{\nabla}_{X} \xi, \xi\right)=-(m-1) \exp (f) \omega(X) .
$$

On the other hand $\omega^{\sharp}$ is orthogonal to $M$. Hence, by the use of (3.13) and (5.2) in the above equation we have $\alpha(X)=0$ for each vector field $X$ on $M$. Thus, by (5.1), the Ricci tensor of $M$ is parallel.

THEOREM 5.2. Let $\dot{M}^{m}(m>2)$ be a submanifold of a conformal Kenmotsu manifold $M$ with recurrent Ricci tensor such that $\omega^{\sharp}$ is normal to $\dot{M}$. Then $\dot{M}$ is an Einstein submanifold.

Proof. It is an immediate result of Theorem 4.1 and Lemma 5.1.

LEMMA 5.3. Let $\dot{M}^{m}(m>1)$, be a submanifold of a conformal Kenmotsu manifold $M$ with recurrent Ricci tensor such that $\omega^{\sharp}$ is tangent to $\dot{M}$. Then

$$
\alpha(X)=\frac{-4(m-1) \exp (f)}{-4(m-1) \exp (f)+(m-2)\left(\left\|\omega^{\sharp}\right\|^{2}-\omega(\xi)^{2}\right)} \omega(X)
$$

for each vector field $X$ tangent to $M$. 
Proof. Suppose that $\omega^{\sharp}$ is parallel on $\bar{M}$. Putting $X=\xi$ in (3.14), we have

$$
\operatorname{Ric}(\xi, \xi)=-(m-1) \exp (f)+\frac{1}{4}(m-2)\left\|\omega^{\sharp}\right\|^{2}-\frac{1}{4}(m-2) \omega(\xi)^{2} .
$$

Taking covariant differentiation of (5.4) along vector field $X$ and using (5.1), we obtain

$$
\begin{aligned}
\alpha(X) \operatorname{Ric}(\xi, \xi)+2 \operatorname{Ric}\left(\dot{\nabla}_{X} \xi, \xi\right)= & -(m-1) \exp (f) \omega(X) \\
& +\frac{1}{4}(m-2) X\left(\left\|\omega^{\sharp}\right\|^{2}\right)-\frac{1}{2}(m-2) \omega(\xi) \omega\left(\dot{\nabla}_{X} \xi\right) .
\end{aligned}
$$

As $\omega^{\sharp}$ is parallel on $\dot{M},\left\|\omega^{\sharp}\right\|^{2}$ is constant on $\dot{M}$. Thus using (3.14), (3.19) and (5.4), we get (5.3).

THEOREM 5.4. Let $\dot{M}^{m}(m>1)$ be a submanifold of a conformal Kenmotsu manifold $M$ with recurrent Ricci tensor such that $\omega^{\sharp}$ is tangent to $\dot{M}^{\prime}$. Then the Ricci tensor of $\dot{M}^{\text {cannot }}$ to be parallel.

Proof. Assuming that the Ricci tensor of $M$ is parallel and using (5.1) we will have

$$
\alpha(X) \operatorname{Ric}(Y, Z)=0 .
$$

Suppose that $\omega^{\sharp}$ is parallel on $\dot{M}$. Taking $Y=Z=\xi$ in the above equation and using (3.14) and (3.19), we get $\alpha=0$. Then from (5.3), we have $\omega^{\sharp}=0$ that is a contradiction.

\section{REFERENCES}

[1] R. Abdi and E. Abedi. Invariant and anti-invariant submanifolds of a conformal Kenmotsu manifold. Azerbaijan J. Math. 5 (2015), 54-63.

[2] R. Abdi and E. Abedi. $C R$-hypersurfaces of a conformal Kenmotsu manifold satisfying certain shape operator conditions. Period. Math. Hungar. 73(1) (2016), 83-92.

[3] A. L. Besse. Einstein Manifolds (Ergebnisse der Mathematik Und ihrer Grenzgebiete. 3. Folge. Bd. 10). Springer, Berlin, 1987.

[4] D. E. Blair. Riemannian Geometry of Contact and Symplectic Manifolds. Birkhäuser, Boston, MA, 2002.

[5] D. E. Blair. Contact Manifolds in Riemannian Geometry (Lecture Notes in Mathematics, 509). Springer, Berlin, 1976.

[6] V. Bonanzinga and K. Matsumoto. Warped product $C R$-submanifolds in locally conformal Kaehler manifolds. Period. Math. Hungar. 48(1-2) (2004), 207-221.

[7] M. C. Chaki and R. K. Maity. On quasi Einstein manifolds. Publ. Math. Debrecen 57(3-4) (2000), 297-306.

[8] R. Deszcz, M. Hotlos and Z. Senturk. On curvature properties of quasi-Einstein hypersurfaces in semiEuclidean spaces. Soochow J. Math. 27 (2001), 375-389.

[9] A. Gray and L. M. Hervella. Sixteen classes of almost Hermitian manifold and their linear invariants. Ann. Math. Pure Appl. 123(3) (1980), 35-58.

[10] Y. Kamishima and L. Ornea. Geometric flow on compact locally conformally Kaehler manifolds. Tôhoku Math. J. 57 (2005), 201-221.

[11] K. Kenmotsu. A class of almost contact Riemannian manifolds. Tôhoku Math. J. 24 (1972), 93-103.

[12] S. Kobayashi and K. Nomizu. Foundations of Differential Geometry. Vol. I. Wiley, New York, 1963.

[13] M. Kobayashi. Semi-invariant submanifolds of a certain class of almost contact manifolds. Tensor (N.S.) 43(1) (1986), 28-36.

[14] N. Papaghiuc. Some remarks on $C R$-submanifolds of a locally conformal Kaehler manifold with parallel Lee form. Publ. Math. Debrecen 43(3-4) (1993), 337-341.

[15] S. S. Shukla and M. K. Shukla. On $\varphi$-Ricci symmetric Kenmotsu manifolds. Novi Sad J. Math. 2 (2008), 89-95.

[16] Y. Wong. Recurrent tensors on a linearly connected differentiable manifold. Trans. Amer. Math. Soc. 99 (1961), 325-341. 


\author{
Roghayeh Abdi \\ Department of Mathematics \\ Azarbaijan Shahid Madani University \\ Tabriz 5375171379 \\ Iran \\ (E-mail: rabdi@azaruniv.ac.ir) \\ Esmaiel Abedi \\ Department of Mathematics \\ Azarbaijan Shahid Madani University \\ Tabriz 5375171379 \\ Iran \\ (E-mail:esabedi@azaruniv.ac.ir)
}

